Article

\title{
Application of the Moment Method for Numerical Simulation of Homogeneous-Heterogeneous Condensation
}

\author{
Igor E. Ivanov ${ }^{1,2}$, Vladislav S. Nazarov ${ }^{1}$ and Igor A. Kryukov ${ }^{3, *(D)}$ \\ 1 Moscow Aviation Institute (National Research University), 4, Volokolamskoe Highway, \\ 125993 Moscow, Russia; ivanovmai@gmail.com (I.E.I.); naz.vladislav@yandex.ru (V.S.N.) \\ 2 Federal State Budget Educational Institution of Higher Education M.V. Lomonosov Moscow State \\ University (Lomonosov MSU), 1, Leninskie Gory, 119991 Moscow, Russia \\ 3 Ishlinsky Institute for Problems in Mechanics of the RAS, 01-1, Pr. Vernadskogo, 119526 Moscow, Russia \\ * Correspondence: ikryukov@gmail.com
}

check for updates

Citation: Ivanov, I.E.; Nazarov, V.S. Kryukov, I.A. Application of the Moment Method for Numerical Simulation of Homogeneous-

Heterogeneous Condensation. Fluids 2022, 7, 68. https://doi.org/10.3390/ fluids7020068

Academic Editor: Mikhail V. Chernyshov

Received: 24 December 2021

Accepted: 4 February 2022

Published: 7 February 2022

Publisher's Note: MDPI stays neutral with regard to jurisdictional claims in published maps and institutional affiliations.

Copyright: (C) 2022 by the authors. Licensee MDPI, Basel, Switzerland. This article is an open access article distributed under the terms and conditions of the Creative Commons Attribution (CC BY) license (https:// creativecommons.org/licenses/by/ $4.0 /)$.

\begin{abstract}
The paper considers the numerical modeling of the processes of homogeneous and heterogeneous condensation and evaporation in multiphase flows using the method of moments. Nonstationary processes of gas dynamics and phase transitions in the two-dimensional plane and axisymmetric regions are described by a general system of equations. The system of equations is expanded by adding two equations. One describes the evolution of the total mass fraction of the condensing substance; the other describes the evolution of the mass fraction of solid particles. An instant wetting model is used to model heterogeneous nucleation. The Gyarmathy model is used for the approximation of the average droplet growth rate. Heterogeneous condensation is modeled based on the distribution function of foreign impurities. An approach to calculating evaporation in the heterogeneous case is proposed. A comparison of the proposed models with a numerical experiment is given. Numerical simulation of homogeneous-heterogeneous condensation in a gas-dynamic ejector is carried out.
\end{abstract}

Keywords: homogeneous condensation; heterogeneous condensation; multiphase flow; method of moments

\section{Introduction}

Gas-dynamic flows with phase transformations are ubiquitous in nature and technology. Condensation of water vapor contained in humid air can play a special role in the flow around aerodynamic surfaces. Condensate generation can affect the performance of steam turbines in power plants. Wet gas flow has a significant effect on the thrust characteristics of rocket engine nozzles. Many chemical processes take place at the phase interface. Using heterogeneous condensation, gas is purified from submicron particles.

In nature, there are two types of vapor condensation. The first type is homogeneous condensation, in which the initial condensation centers (nuclei) arise spontaneously from the vapors of the substance itself [1-9]; the second type is heterogeneous condensation, when particles of another (usually solid) phase are the centers of condensation [10-16]. Condensation of a binary gas mixture, in which vapors of one substance can condense on the nuclei of another substance [17], is a special case of heterogeneous condensation. Heterogeneous nucleation associated with the initial adhesion of clusters to the surface of particles of another phase will be called activation.

The process of both homogeneous and heterogeneous condensation can be divided into two stages: the first is nucleation, and the second is the growth of droplets due to condensation. At the first stage, the majority of stable nuclei of a new phase are formed from the initial metastable phase (supersaturated vapor). The nucleation of a new phase occurs due to the high degree of metastability of the medium, and the end of the nucleation process is due to a decrease in the degree of metastability due to the transition of a part 
of the substance of the metastable phase into nuclei of a stable phase. At the stage of condensation growth of droplets, the size of droplets (nuclei) of a new phase increases with a constant number of droplets.

Depending on the role of other phase particles, nucleation can occur either through homogeneous nucleation or through heterogeneous activation.

Heterogeneous nucleation is determined by such factors as the size distribution of solid condensation nuclei and their concentration, the concentration of vapor monomers at the core surface, and the activation energy of nucleation, which depends largely on the contact angle of wetting of the core surface liquid. Because there is lower energy consumption during activation than during homogeneous condensation, heterogeneous condensation leads to less supercooling of the mixture and a faster approach to equilibrium. It can be assumed that if the probability of nucleation is higher near foreign bodies than in other parts of the medium, then the condensation process proceeds according to a heterogeneous type. However, if there are not enough foreign particles (or if they are absent), then condensation is homogeneous during the transition from a metastable state to a stable. As a rule, heterogeneous nucleation occurs at earlier stages of undercooling (at lower parameters characterizing the metastability of the medium) than homogeneous nucleation. However, in many natural phenomena and some technical devices, due to the different flow conditions in the flow, the joint occurrence of both condensation processes is possible.

In recent years, the problems of the numerical modeling of homogeneous [18-25] and heterogeneous condensation [26-28] have been actively studied. The problem of the joint occurrence of heterogeneous and homogeneous nucleation in solutions and melts was considered in [29], and in gas mixtures in [30].

In the equation for the nucleation rate obtained in the classical heterogeneous nucleation theories [31-33], a coefficient is introduced into the exponential function. This coefficient is defined as an interfacial correlation function $f$. With this approach, previously proposed in [34], the free energy of the formation of the critical embryo on the nucleating particle at a given supersaturation is defined as the product of the free energy barrier of homogeneous nucleation and the interphase correlation function $f$. Heterogeneous nucleation corresponds to low values of $f$ and small values of supersaturation, homogeneous nucleation corresponds to high values of supersaturation and $f=1$. The $f$ value is determined by the interfacial tension between different phases and the average radius of the foreign particles divided by the critical radius of the embryos.

The dynamics of the condensation process are described by the general dynamic equation (GDE) [20], which sets the law of evolution of the size distribution function of liquid phase droplets. Solving this equation requires significant computational resources to store and work with multidimensional data sets. One of the most widely used approaches to solving GDE is the transition from a differential equation for the distribution function to a system of integrodifferential equations for the moments of this distribution function. The resulting system of equations requires closure. Hill [18] was one of the first to propose a practical model based on the method of moments (MM). He proposed a form of moment equations that was applicable to any level of droplet growth by approximating the average droplet growth rate. The moment method was further developed in [21,22].

From the point of view of a mathematical description, heterogeneous nucleation (activation) differs from homogeneous nucleation by a function that sets the rate of nucleation. For condensation, the deposition of vapors on foreign clusters is energetically more preferable than the formation of new ones of their own. Fletcher was one of the first to propose the form of the nucleation rate function for spherical particles [34]. This approach is preferable for considering heterogeneous cluster formation on relatively large particles. An alternative approach is an assumption that the particles are wetted entirely at once. This approach is called the instantaneous-wetting model [28] and gives good results for droplets of the order of tens of nanometers. The model makes it easy to adapt MM for heterogeneous condensation. 
For the closure of the system of moment equations in $\mathrm{MM}$, it is essential to assume that the droplet growth rate has a constant or linear dependence on the radius. With more complex dependences of the droplet growth rate on the radius, the use of MM is difficult. To eliminate this drawback of MM in 1997, McGraw [21] proposed a modification of MM, the quadrature method of moments (QMOM), with which the moments of the distribution function are tracked in time in the same way as in the usual case, but for which the exact closure requirement is replaced by the approximate closure, which allows the method to be applied to a much wider range of cases.

In 2011, Yuan and Fox [35] published the conditional QMOM (CQMOM) method. This method is more common and is applicable for modeling general problems by tracking the moments of the droplet size distribution function with an arbitrary number of internal parameters.

The efficiency of QMOM was studied in [36,37] when studying the process of primary and secondary nucleation in the transonic flow in a low-pressure steam turbine. The stability of QMOM is shown in the case of the calculation of condensation processes for a polydisperse distribution of droplets with sizes differing by several orders of magnitude.

In most of the computational cases considered in this work, droplets grow in the free molecular regime. Therefore, we assume that the droplet growth rate does not depend on the droplet radius [21] and apply the standard MM approach.

In this work, we propose a mathematical model for studying the joint occurrence of heterogeneous and homogeneous condensation processes in technological devices. An approach based on the use of the moment method for modeling homogeneous and heterogeneous condensation is considered and supplements the study [26] in part of the model of heterogeneous evaporation. In this paper, we propose to use the initial distribution function of dry particles during denucleation and to perform denucleation when the size distribution function of droplets (wetted particles) reaches the initial distribution function of dry particles. This distinguishes our model from previous works [25,38,39], in which the critical drop nucleus was used for evaporation.

\section{Physical and Mathematical Models}

Often in applications, problems arise when homogeneous and heterogeneous condensation is realized simultaneously in gas-dynamic flows [40]. In cases where the degree of supersaturation $S$ has very large values [29,30], homogeneous condensation prevails in the medium. The embryos are formed mainly from vapor molecules even when the number of foreign particles is insufficient for the heterogeneous condensation.

Unlike homogeneous condensation, heterogeneous condensation occurs on alreadyexisting impurities: drops of another already-condensed gas, soot, or other small particles that act as condensation nuclei. For homogeneous condensation, we consider the condensation process as having two successive stages: nucleation and droplet growth. However, in contrast to homogeneous condensation, heterogeneous nucleation occurs due to an increase in the number of nuclei into which stable clusters of a condensing liquid, with a size greater than the critical size, nucleate (attach).

The physical model in this study is a multiphase medium consisting of a multicomponent gas (carrier gas/air and vapors of a condensing substance/water), and clusters (drops) of a condensing substance and solid foreign particles.

When constructing a mathematical model of the flow of a multiphase mixture with phase transitions, we use the following assumptions:

(1) Volume fractions of liquid and solid phases are negligible.

(2) Homogeneous droplets are in mechanical and thermal equilibrium with the gas phase. There is only mechanical equilibrium between heterogeneous droplets and the gas phase, that is, they have their own temperature.

(3) There are no collisions between drops.

(4) Diffusion between the vapors of the condensing substance and the carrier gas are neglected in the equations for the mass fractions of the condensing substance. 
In addition, in our model, we suppose:

(1) Condensation occurs in two stages. As in the homogeneous case, the first is the nucleation stage, the second is the growth of droplets;

(2) The solid particle is completely covered with moisture at once (Figure 1);

(3) The particle size distribution function for solid particles is known in advance and does not change;

(4) The growth of the droplet is uniform in all directions.

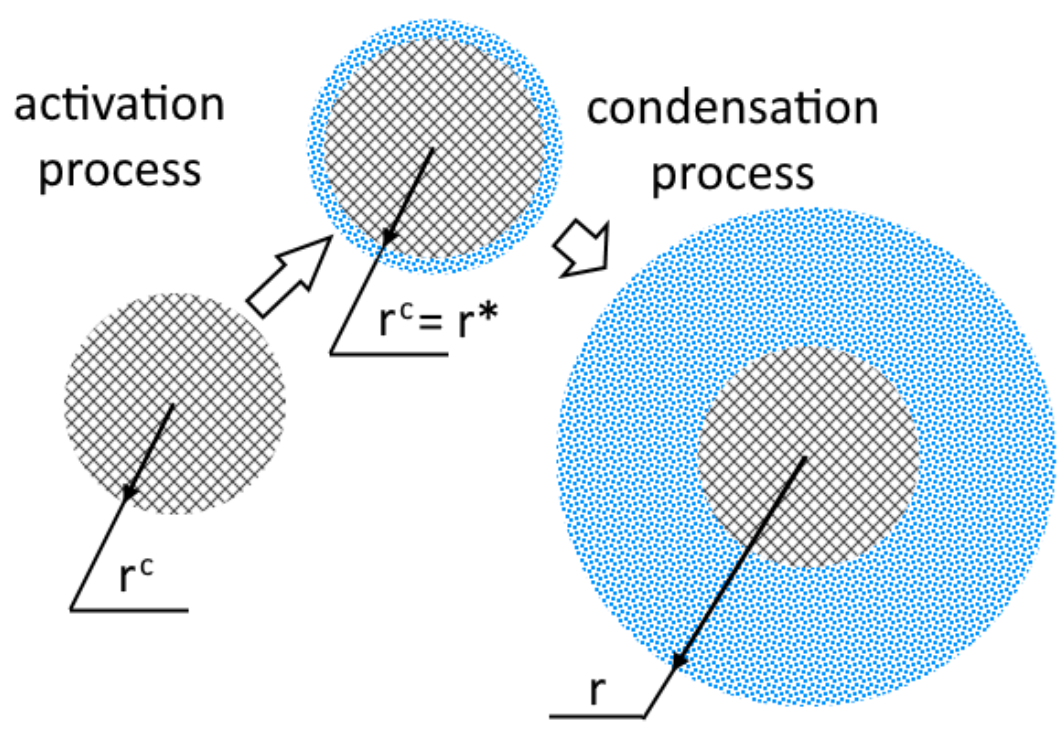

Figure 1. Schematic representation of the heterogeneous condensation process, inspired by [26].

Furthermore, we assume that the activation of particles occurs only for particles of which the radius is greater than the critical radius of the nucleus $r^{*}$ (Figure 2) and that the rest of the particles do not participate in the process. Figure 2 shows the particle size distribution function $g(r)$. The arrow indicates the direction of propagation of the boundary of the activated particle with increasing supersaturation.

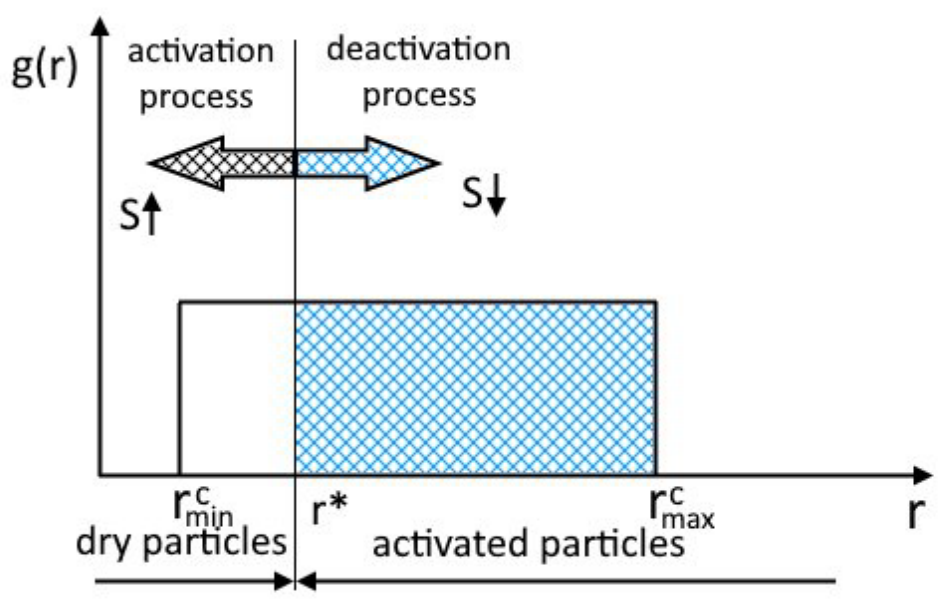

Figure 2. Schematic representation of particle activation, inspired by [26].

To describe the model of homogeneous-heterogeneous condensation, we assume that homogeneous condensation occurs only when the number of formed heterogeneous clusters is insufficient for the medium to pass from a metastable state to a stable one, only due to the formation of heterogeneous nuclei. 


\subsection{Model of Homogeneous-Heterogeneous Condensation}

Within the framework of this approach, we use a system of equations that describes the dynamics of a viscous heat-conducting gas-droplet medium and an extended system of moment equations that describes phase transitions. Additionally, in this model, it is supposed that phase transitions according to homogeneous and heterogeneous types occur independently and therefore the system of moment equations can be represented as a combination of two systems of moment equations:

$$
\begin{aligned}
& \frac{\partial \rho}{\partial t}+\frac{\partial(\rho u)}{\partial x}+\frac{\partial(\rho v)}{\partial y}=-\frac{\xi \rho v}{y} \\
& \frac{\partial(\rho u)}{\partial t}+\frac{\partial\left(\rho u^{2}+p-\tau_{x x}\right)}{\partial x}+\frac{\partial\left(\rho u v-\tau_{x y}\right)}{\partial y}=-\frac{\xi}{y}\left(\rho u v-\tau_{x y}\right), \\
& \frac{\partial(\rho v)}{\partial t}+\frac{\partial\left(\rho u v-\tau_{y x}\right)}{\partial x}+\frac{\partial\left(\rho v^{2}+p-\tau_{y y}\right)}{\partial y}=-\frac{\xi}{y}\left(\rho v^{2}-\tau_{y y}\right), \\
& \frac{\partial(\rho E)}{\partial t}+\frac{\partial\left(u(\rho E+p)-\left(u \tau_{x x}+v \tau_{y x}-q_{x}\right)\right)}{\partial x}+\frac{\partial\left(v(\rho E+p)-\left(u \tau_{y x}+v \tau_{y y}-q_{y}\right)\right)}{\partial y}=, \\
& -\xi \frac{v(\rho E+p)-\left(u \tau_{y x}+v \tau_{y y}-q_{y}\right)}{y} \\
& \frac{\partial}{\partial t}\left(\rho Q_{0}^{h o}\right)+\frac{\partial}{\partial x}\left(\rho u Q_{0}^{h o}\right)+\frac{\partial}{\partial y}\left(\rho v Q_{0}^{h o}\right)=J-\frac{\xi \rho v Q_{0}^{h o}}{y}, \\
& \frac{\partial}{\partial t}\left(\rho Q_{1}^{h o}\right)+\frac{\partial}{\partial x}\left(\rho u Q_{1}^{h o}\right)+\frac{\partial}{\partial y}\left(\rho v Q_{1}^{h o}\right)=r_{*} J+\rho Q_{0}^{h o} \dot{r}-\frac{\xi \rho v Q_{1}^{h o}}{y}, \\
& \frac{\partial}{\partial t}\left(\rho Q_{2}^{h o}\right)+\frac{\partial}{\partial x}\left(\rho u Q_{2}^{h o}\right)+\frac{\partial}{\partial y}\left(\rho v Q_{2}^{h o}\right)=r_{*}^{2} J+2 \rho Q_{1}^{h o} \dot{r}-\frac{\xi \rho v Q_{2}^{h o}}{y}, \\
& \frac{\partial\left(\rho \alpha^{h o}\right)}{\partial t}+\frac{\partial\left(\rho u \alpha^{h o}\right)}{\partial x}+\frac{\partial\left(\rho v \alpha^{h o}\right)}{\partial y}=\frac{4}{3} \pi \rho_{l}\left(r_{*}^{3} J+3 \rho Q_{2}^{h o} \dot{r}\right)-\frac{\xi \rho v \alpha^{h o}}{y}, \\
& \frac{\partial}{\partial t}\left(\rho Q_{0}^{h e}\right)+\frac{\partial}{\partial x}\left(\rho u Q_{0}^{h e}\right)+\frac{\partial}{\partial y}\left(\rho v Q_{0}^{h e}\right)=\psi_{0}-\frac{\xi \rho v Q_{0}^{h e}}{y}, \\
& \frac{\partial}{\partial t}\left(\rho Q_{1}^{h e}\right)+\frac{\partial}{\partial x}\left(\rho u Q_{1}^{h e}\right)+\frac{\partial}{\partial y}\left(\rho v Q_{1}^{h e}\right)=\psi_{1}+\rho Q_{0}^{h e} \dot{r}-\frac{\xi \rho v Q_{1}^{h e}}{y}, \\
& \frac{\partial}{\partial t}\left(\rho Q_{2}^{h e}\right)+\frac{\partial}{\partial x}\left(\rho u Q_{2}^{h e}\right)+\frac{\partial}{\partial y}\left(\rho v Q_{2}^{h e}\right)=\psi_{2}+2 \rho Q_{1}^{h e} \dot{r}-\frac{\xi \rho v Q_{2}^{h e}}{y}, \\
& \frac{\partial\left(\rho \alpha^{h e}\right)}{\partial t}+\frac{\partial\left(\rho u \alpha^{h e}\right)}{\partial x}+\frac{\partial\left(\rho v \alpha^{h e}\right)}{\partial y}=\frac{4}{3} \pi \rho_{l} \rho Q_{2}^{h e} \dot{r}-\frac{\xi \rho v \alpha^{h e}}{y}, \\
& \frac{\partial\left(\rho \alpha_{\max }\right)}{\partial t}+\frac{\partial\left(\rho u \alpha_{\max }\right)}{\partial x}+\frac{\partial\left(\rho v \alpha_{\max }\right)}{\partial y}=-\frac{\xi \rho \alpha_{\max } v}{y}, \\
& \frac{\partial\left(\ell_{\max }\right)}{\partial t}+\frac{\partial\left(\rho u \ell_{\max }\right)}{\partial x}+\frac{\partial\left(\rho v \ell_{\max }\right)}{\partial y}=-\frac{\xi \rho \ell_{\max } v}{y} \\
& \tau_{x x}=\frac{2}{3} \mu\left[2 \frac{\partial u}{\partial x}-\frac{\partial v}{\partial y}-\xi \frac{v}{y}\right] \\
& \tau_{y y}=\frac{2}{3} \mu\left[2 \frac{\partial v}{\partial y}-\frac{\partial u}{\partial y}-\xi \frac{v}{y}\right], \tau_{x y}=\tau_{y x}=\mu\left[\frac{\partial u}{\partial y}+\frac{\partial v}{\partial x}\right], \\
& q_{x}=-\lambda \frac{\partial T}{\partial x}, q_{y}=-\lambda \frac{\partial T}{\partial y} .
\end{aligned}
$$


Here $O x y$ is the cylindrical coordinate system; $\rho$ is the density of the mixture; $p$ is the pressure; $T$ is the static temperature of the mixture; $u$ is the velocity along the $x$ direction; $v$ is the velocity along the $y$ direction; $E$ is the total energy per unit mass; $\mu$ is the viscosity coefficient; $\lambda$ is the thermal conductivity coefficient; $Q_{0}^{h o}, Q_{1}^{h o}, Q_{2}^{h o}$ are the moments of the size distribution function of homogeneous droplets $\left(Q_{0}^{\text {ho }}\right.$ - the number of clusters (drops), $Q_{1}^{h o}$ - the sum of the radii of all drops, $Q_{2}^{\text {ho }}$ - the sum of the squares of the radii of all drops in a kilogram of the mixture); $Q_{0}^{h e}, Q_{1}^{h e}, Q_{2}^{h e}$ are the moments of the distribution function of heterogeneous drops by size; $\alpha^{h o}$ is the mass fraction of water condensed in a homogeneous way (the mass of liquid water in a kilogram of the mixture); $\alpha^{\text {he }}$ is the mass fraction of water condensed in a heterogeneous way; $J^{\text {ho }}$ is the rate of homogeneous nucleation (the number of nuclei of critical size formed in a cubic meter per unit of time); $r_{*}$ is the critical nucleus radius, $\dot{r}=d r / d t$ is the rate of droplet growth; $\tau_{x x}, \tau_{x y}, \tau_{y y}$ are the components of the viscous stress tensor; and $q_{x}, q_{y}$ are the components of the heat flux. In the plane case, $\xi$ is 0 , and in the axisymmetric coordinate system, $\xi$ is 1 .

Effective viscosity and thermal conductivity are defined as the sum of the laminar $\mu_{L}$, $\lambda_{L}$ and turbulent $\mu_{t}, \lambda_{t}$ components:

$$
\mu=\mu_{L}+\mu_{t}, \lambda=\lambda_{L}+\lambda_{t}
$$

To calculate the turbulent viscosity and thermal conductivity, an algebraic turbulence model [41] is used.

Heterogeneous activation affects homogeneous nucleation through the terms contained in the right-hand sides of Equations (5)-(8):

$$
J=\left\{\begin{array}{c}
\eta\left(J^{h o}-\psi_{0}^{h e}\right), S>1 \\
J_{\text {den }}^{h o} S<1
\end{array},\right.
$$

where $\psi_{0}^{\text {he }}$ is the rate of heterogeneous activation (the number of particles of the solid phase activated in a cubic meter per second), determined [26] on the assumption that the existence of droplets less than the critical size is energetically unfavorable:

$$
\psi_{k}^{h e}=\frac{d}{d t} \int_{0}^{\infty} r^{k} \rho g d r
$$

If the rate $J^{h o}$ is less than $\psi_{0}^{h e}$, homogeneous nucleation does not occur; however, otherwise both the presence of homogeneous and heterogeneous condensation is possible. This condition is taken into account in Equations (5)-(8) using the coefficient $\eta$, calculated from the value of $J$ from (19):

$$
\eta=\left\{\begin{array}{l}
1, \text { if } J>0 \\
0, \text { if } J<0
\end{array} .\right.
$$

In Equations (9)-(12), the terms $\psi_{k}$ on the right-hand sides, describing activation upon condensation and deactivation upon evaporation of heterogeneous droplets, are written as follows:

$$
\psi_{k}=\left\{\begin{array}{c}
\psi_{k}^{h e}, S>1 \\
\psi_{\text {deactiv }}^{h e}, k=0 \\
0
\end{array}, S<1 ，\right.
$$

Here the value $\psi_{\text {deactiv }}^{\text {he }}$ is the deactivation rate.

For the case of a uniform distribution function of $g_{n}$ foreign particles, with a particle size distribution from $r_{\min }$ to $r_{\max }$, the rate of heterogeneous activation will have the following form:

$$
\psi_{k}^{h e}=\left\{\begin{array}{c}
0, \quad r_{*} \notin\left[r_{\min }, r_{\max }\right] \\
-\frac{\rho g_{n}}{r_{\max }-r_{\min }} r_{*}^{k} \frac{d r_{*}}{d t}, \quad r_{*} \in\left[r_{\min }, r_{\max }\right]
\end{array},\right.
$$

where $g_{n}$ is the total number of particles per unit mass for a dry mixture. 
To calculate the derivative $d r_{*} / d t$, we used the values $r_{*}$ on the current $k$ and the previous $k-1$ layers:

$$
\frac{d r_{*}}{d t}=\frac{r_{*}^{k}-r_{*}^{k-1}}{d t}
$$

It should be noted that with an increase in the supersaturation $S$, the radius $r_{*}$ decreases and, as a result, the derivative will have a negative sign.

The physical model of the medium is a mixture of five components: a carrier gas, a vapor of a condensing substance and homogeneous drops of a condensing substance, heterogeneous drops of a condensing substance, and foreign solid particles. Quantitatively, each component is characterized by its mass fraction, that is, the ratio of the mass of the component in a kilogram of the mixture to the kilogram of the mixture, so that the sum of the mass fractions of all components is equal to one. For a correct representation of material balances (conservation laws) in a multiphase medium, it is necessary to use five equations in a mathematical model that describe the evolution in time and space of the mass fractions of five components. Other representations are also possible, for example, one equation describing the evolution of the mixture density (the continuity equation) and four equations for describing the evolution of the mass fractions of four components. The mixture density is defined here as the ratio of a kilogram of the mixture (the sum of the mass fractions of the components multiplied by the kilogram) to the volume occupied by this mixture. In this work, the general system of equations contains:

- $\quad$ continuity Equation (1) (for the mixture density);

- $\quad$ Equation (8) for describing the evolution of the mass fraction of the liquid phase $\alpha^{\text {ho }}$ in homogeneous droplets;

- $\quad$ Equation (13) to describe the evolution of the mass fraction of the condensing fraction $\alpha_{\max }$ (that is, the sum of the mass fractions of the liquid fraction in homogeneous and heterogeneous droplets and the vapor fraction $\alpha_{\max }=\alpha^{h o}+\alpha^{h e}+\alpha_{\text {vap }}$, where $\alpha_{\text {vap }}$ is the mass fraction of the vapor of the condensing substance-water);

- Equation (12) for describing the evolution of the mass fraction of the liquid phase $\alpha^{\text {he }}$ in heterogeneous droplets; and

- Equation (14) for describing the evolution of the mass fraction of the solid phase $\ell_{\max }$.

Thus, for a correct description of the inhomogeneity of a multiphase medium, the equation for the propagation of the mass fraction of the condensing phase in homogeneous condensation (13) and the propagation of the mass fraction of the solid phase (14) was introduced. The addition of these equations to the general system (1)-(22) makes it possible to expand the class of flows with condensation that can be calculated. For example, this allows us to consider problems in which, in the initial distribution of parameters in different zones in the computational domain, different content of the condensed matter $\alpha_{\max }$ and solid particles $\ell_{\max }$ is specified, or the flow in channels with a time-varying value of $\alpha_{\max }$ and $\ell_{\max }$ on input.

Since the system of basic Equations (1)-(22) implements the assumption that different types of condensation occur separately, which can lead to a situation in which the reconstructed particle size distribution function will have a bimodal form. Both condensation processes proceed with different intensities and can be separated in time.

\subsection{Determination of the Parameters of the Homogeneous Nucleation}

In $[18,31-34,42]$, the dependence of the nucleation function on correcting factors that take into account the curvature of the drop, the stationarity of the process, and the onset of the nucleation process was obtained:

$$
J=\frac{\beta}{(1+\eta)} \sqrt{\frac{2 \sigma}{\pi m^{3}}} \frac{\rho_{v}^{2}}{\rho_{l}} \exp \left(-b \frac{4 \pi}{3} \frac{r_{*}^{2} \sigma}{R_{V} m T}\right),
$$


where $1 /(1+\eta)$ is a correction factor introduced to take into account the nonstationarity of the process [43],

$$
\eta=2 \frac{\gamma_{f}-1}{\gamma_{f}+1} \frac{L}{R_{v} T}\left(\frac{L}{R_{v} T}-\frac{1}{2}\right),
$$

$\beta$ is the condensation coefficient; $\sigma=k_{\sigma} \sigma_{\infty} ; \sigma_{\infty}$ is the surface tension of a flat film; $k_{\sigma}$ is a correction factor that takes into account the curvature of the drop; $b$ is a factor correcting the onset of nucleation; $S=p_{V} / p_{S}$ is the supersaturation parameter; $T$ is the temperature of the medium; $L$ is the specific heat of condensation; $\gamma_{f}$ is the isentrope of the mixture; $R_{v}$ is the gas constant for water vapor; $R$ is the universal gas constant; $\rho_{l}$ is the density of the liquid phase; and $m$ is the mass of the molecule of the condensing substance (water).

It is assumed that only those particles are activated which have a radius less than the critical radius of the particles $r_{*}$, which is found from the condition of the maximum of the thermodynamic barrier:

$$
r_{*}=\frac{2 \sigma}{R T \rho_{l} \ln S} .
$$

\subsection{The Growth Rate of Homogeneous Droplets}

Two different models are used to determine the growth rate of homogeneous and heterogeneous droplets. To calculate the growth rate of homogeneous droplets, the HertzKnudsen model is used:

$$
\frac{d r}{d t}=\frac{\beta}{\rho_{l}} \frac{p_{V}-p_{S, r}}{\sqrt{2 \pi R_{V} T}}
$$

$p_{S, r}=p_{S} \exp \left(2 \sigma /\left(\rho_{l} R_{V} T r_{\text {Hill }}\right)\right)$ is the saturation pressure on the surface of a drop of average size radius. $\beta$ is the condensation coefficient.

The Gyarmathy model is used to calculate the growth rate of heterogeneous droplets [44]:

$$
\frac{d r^{2}}{d t}=\frac{N u_{\dot{H}} \lambda}{\rho_{l} L}\left(T_{d}-T\right),
$$

where $N u_{\dot{H}}$ is the Nusselt number for the heat flux, $T_{d}$ is the droplet temperature, and $L$ is the specific heat of condensation.

To find the droplet temperature $T_{d}$, it is necessary to use implicit relations (27). The implicit computation of temperature is a very computationally expensive procedure. However, there is an approximate explicit formula for the calculation, which has a small error at a small saturation ratio [42]:

$$
\left(\frac{T_{d}}{T}-1\right)=f\left(S, K e_{\infty}\right)\left[C_{1}+C_{2}\right]^{-1}\left(1-\delta_{1}\right),
$$

where

$$
\begin{gathered}
K e=\frac{2 \sigma}{\rho_{l} R_{v} T r}, \delta_{1} \approx \frac{0.5 C_{1}^{2}-C_{2}}{\left(C_{1}+C_{2}\right)^{2}}\left(\ln S-K e_{\infty}\right), \delta_{1} \approx \frac{0.5 C_{1}^{2}-C_{2}}{\left(C_{1}+C_{2}\right)^{2}}\left(\ln S-K e_{\infty}\right), \\
C_{1}=\frac{T}{\theta S}\left(\frac{p}{p_{s}}-S\right), C_{2}=\frac{L}{R_{v} T},
\end{gathered}
$$

where the coefficient $\theta$ is calculated from the following expression:

$$
\theta=\frac{D_{\bmod } L N u_{\dot{M}}}{k_{m} N u_{\dot{E}}},
$$

$D_{\text {mod }}$ is the modified diffusion coefficient equal to $D_{\bmod }=D_{m} p /\left(R_{v} T_{m}\right)$, where $D_{m}=2.54(T / 295)^{2.085} / p$ is for water. 
For the Nusselt numbers $N u_{\dot{M}^{\prime}} N u_{\dot{H}^{\prime}} N u_{\dot{E}}$ the following relations are valid:

$$
\begin{gathered}
N u_{\dot{E}}=N u_{\dot{H}}=\frac{N u_{\dot{H}}^{c t}}{1+\left(N u_{\dot{H}}^{c t} / B_{\dot{H}}\right) K n}, \\
N u_{\dot{M}}=\frac{N u_{\dot{M}}^{c t}}{1+\left(N u_{\dot{M}}^{c t} / B_{\dot{M}}\right) K n} .
\end{gathered}
$$

Smolders [28] proposed an approximate version of formulas (29) and (30) in the case of water condensation and selected for the continuous Nusselt numbers $N u_{\dot{M}}^{c t}=N u_{\dot{H}}^{c t}=2$, the values of $B_{\dot{H}}=0.49$ and $B_{\dot{M}}=0.62$.

\subsection{Closing Relations}

We assume that the mixture is in thermodynamic equilibrium. In contrast to the cases of homogeneous and heterogeneous condensation, the combined model takes into account the presence of the mass fraction of the solid fraction in the flow of the multiphase mixture. The thermodynamic parameters of the mixture are written as follows:

$$
\begin{gathered}
C_{V \text { mixt }}=\left(1-\alpha_{\max }-l_{\max }\right) C_{V a}+\alpha_{\max } C_{V V}+\alpha\left(C_{l}-C_{V V}\right)+l_{\max } C_{s}, \\
C_{P \text { mixt }}=\left(1-\alpha_{\max }-l_{\max }\right) C_{P a}+\alpha_{\max } C_{P V}+\alpha\left(C_{l}-C_{P V}\right)+l_{\max } C_{s}, \\
R_{\text {mixt }}=\left(1-\alpha_{\max }\right) R_{a}+\alpha_{\max } R_{V}-\alpha R_{V}, \gamma_{f}=C_{P_{\text {mixt }}} / C_{V \text { mixt }},
\end{gathered}
$$

where $C_{s}$ is the specific heat capacity of the solid fraction; $C_{V a}, C_{P a}$ are the specific heat capacities at constant volume and constant pressure for the carrier gas; $C_{V V}, C_{P V}$ are the specific heat capacities for vapors of the condensing substance; $C_{V \text { mixt }}, C_{P \text { mixt }}$ are the specific heat capacities for a two-phase mixture; $C_{l}$ is the specific heat for the liquid; and $R_{a}, R_{V}, R_{\text {mixt }}$ are gas constants of the carrier gas, vapors of the condensing substance, and the two-phase mixture.

The caloric and thermal equations of state are as follows:

$$
\begin{gathered}
T=\frac{\left(E-u^{2} / 2\right)+\alpha L_{0}}{\left(1-\alpha_{\max }-l_{\max }\right) C_{V a}+\alpha_{\max } C_{V V}+\alpha\left(C_{l}-C_{V V}\right)+l_{\max } C_{s}}, \\
p=\rho T R_{\text {mixt }}, \\
C_{f}^{2}=\gamma_{f} p / \rho, L=L_{1} T+L_{0}, L_{1}=C_{P V}-C_{l},
\end{gathered}
$$

where $C_{f}$ is the speed of sound.

The mass fraction of water is calculated as the sum of the mass fractions of liquid $\alpha$ formed in the homogeneous case and the heterogeneous case:

$$
\alpha=\alpha^{h o}+\alpha^{h e} .
$$

The molecular viscosity is calculated using the Sutherland law:

$$
\mu_{l}=\mu_{0}\left(\frac{T}{T_{0}}\right)^{3 / 2} \frac{T_{0}+C}{T+C},
$$

where $\mu_{0}=1.75 \times 10^{-5}$ is the dynamic viscosity for $T_{0}=273 \mathrm{~K}, \mathrm{C}=122 \mathrm{~K}$.

The rates of heterogeneous nucleation (activation) and denuclearization (deactivation) depend on the concentration of particles in the mixture; therefore, to calculate these values, it is necessary to know the mass concentration of foreign particles at each point of the computational domain. 


\subsection{Modeling Heterogeneous Evaporation and Denucleation}

Evaporation occurs at $S<1$. In this case $d r / d t<0$ and the droplet radius decreases. We assume that the deactivation of particles (their complete drying) begins when the droplet size reaches the initial values of the size of solid particles. Figure 3 illustrates the processes of evaporation and deactivation using the example of a uniform distribution of foreign particles.
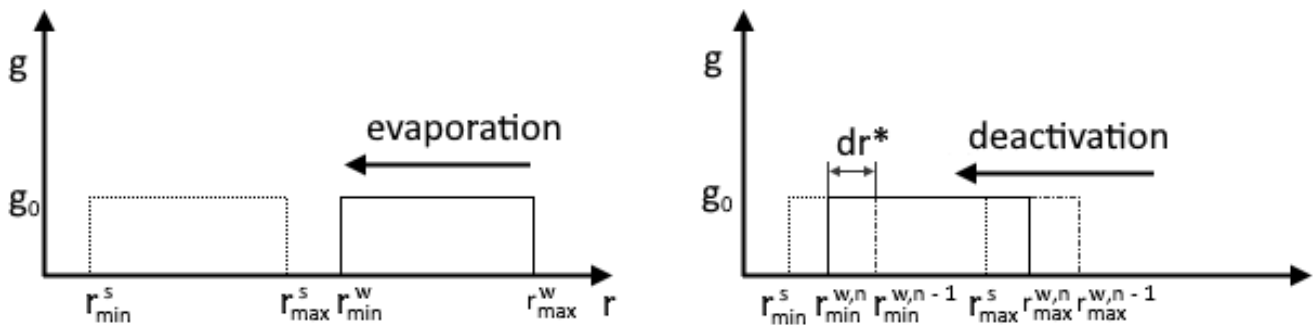

Figure 3. Comparison of evaporation and deactivation processes.

The deactivation process starts when the minimum radius of particles covered with a water film reaches the maximum radius of the spectrum of solid foreign particles. The particle deactivation rate is determined according to the ratio:

$$
J_{\text {deactiv }}=\frac{g_{0}}{r_{\max }^{w}-r_{\min }^{w}} \frac{\mathrm{d} r_{d a}^{*}}{\mathrm{~d} t} .
$$

The main difference between the deactivation model (heterogeneous denuclearization) and the model proposed by Luo $[26,38]$ is the calculation of the derivative of the droplet growth rate. To calculate this, the values of the minimum particle radii at the $n$ and $n-1$ layers are used:

$$
\frac{\mathrm{d} r_{\mathrm{da}}^{*}}{\mathrm{~d} t}=\frac{r_{\min }^{n}-r_{\min }^{n-1}}{\mathrm{~d} t} .
$$

\section{Numerical Method}

The system of Equations (1)-(14) is solved by means of the numerical method [23-25]. It is based on the Godunov high-resolution method and uses the MUSCL approach to improve the spatial accuracy of the method. The second-order accuracy in space for smooth solutions is achieved using a linear reconstruction for the primitive variables within each computational cell. The use of slope limiters ensures the monotonicity of the obtained solutions.

The fluxes through the faces of the computational cells are calculated using an approximate solution to the Riemann problem (HLL or AUSM+).

The viscous and diffusion terms in (1)-(14) are discretized using the control volume scheme, which on a uniform Cartesian grid is reduced to the standard central difference approximation of the second derivatives. All source terms are explicitly approximated. To advance the calculation along the time coordinate, the explicit Euler method is used.

The algorithm is implemented for two-dimensional regions of complex geometric shapes. To approximate the system of equations, we introduce a regular computational grid consisting of convex quadrilateral cells and adapt them to the boundaries of the region.

\section{The Algorithm Testing}

The developed mathematical model and computational algorithm were tested by solving problems on the flow of moist air (nitrogen) with purely homogeneous condensation and with heterogeneous condensation and evaporation. 


\subsection{Homogeneous Condensation of Water Vapor in a Laval Nozzle}

The experimental conditions from [43] are considered as the first test case. In this experiment, the condensation of water vapor in nitrogen was investigated in a plane nozzle. Following [43], the pressure in the receiver is set to be equal to $60,000 \mathrm{~Pa}$, the temperature is $287^{\circ} \mathrm{K}$ and the partial pressure of water vapor is $1000 \mathrm{~Pa}$ (the mass fraction of water vapor is $\alpha_{\max }=0.11$ ). The origin of the coordinate system is located at the center of the nozzle throat. The computational domain is shown in Figure 4, and the results of calculations are compared with the experiment [43] in Figure 5.

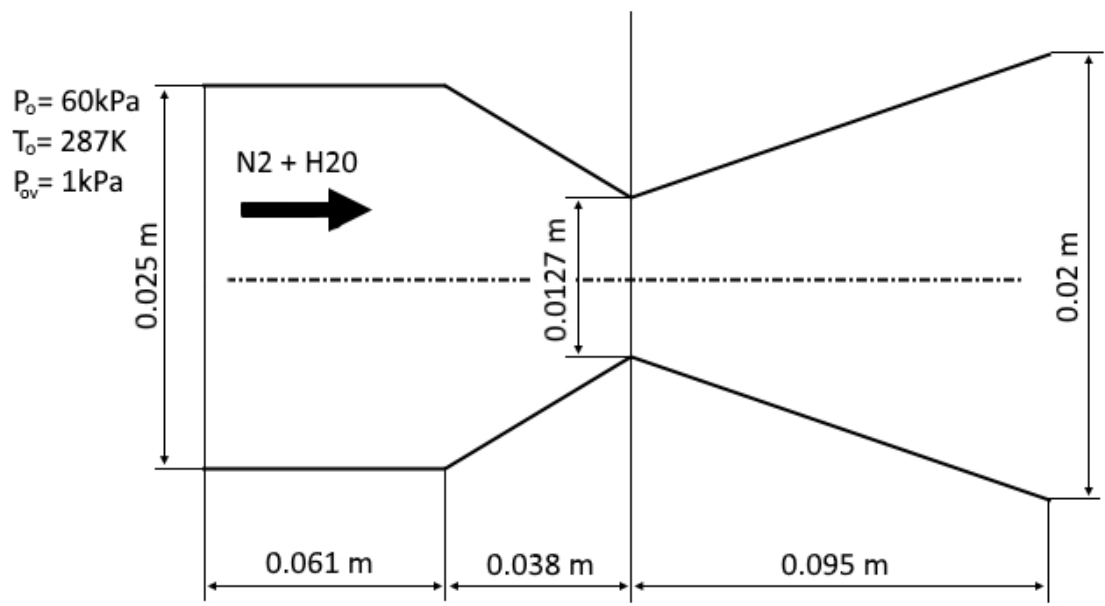

Figure 4. Computational domain for the plane nozzle.

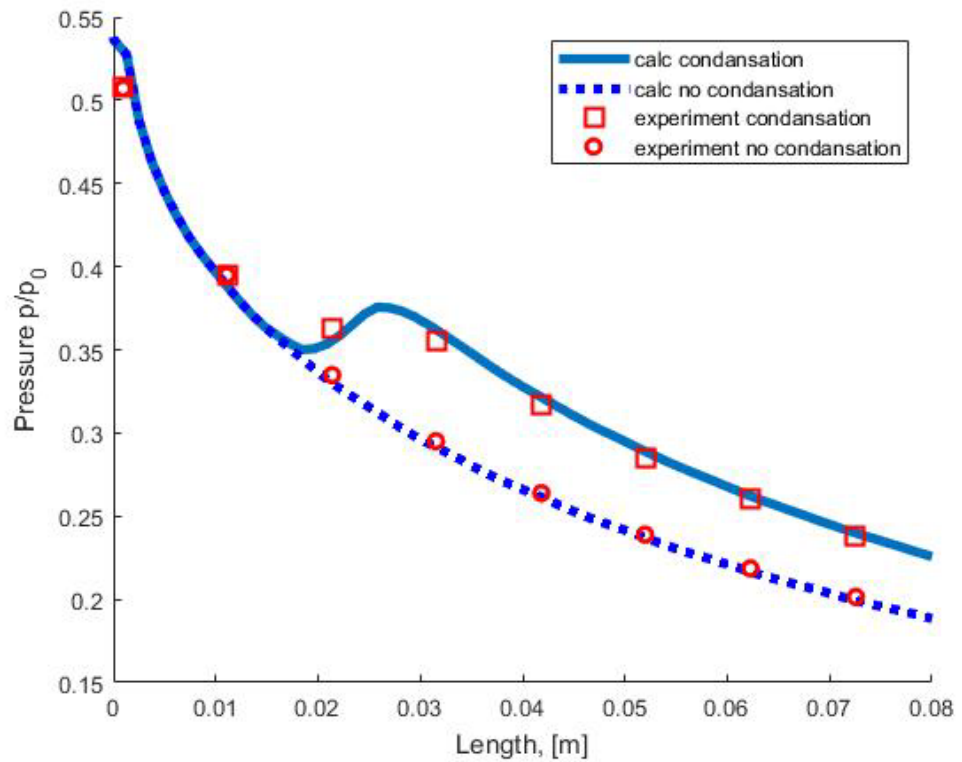

Figure 5. Pressure distribution along the nozzle axis.

Figure 5 shows a comparison of the pressure distribution in the nozzle with experimental data from [43]. Symbols (circles and squares) correspond to the experimentally measured pressure on the nozzle wall in an adiabatic (without condensation) flow and in a flow with condensation. The dotted line corresponds to the results of calculating the adiabatic flow. The solid line corresponds to the calculation of the flow with condensation using the moment method.

Analysis of the calculation results and comparison with experimental data in a wide range of partial pressures of water vapor at the nozzle inlet show that the moment method correctly predicts the onset of nucleation and takes into account the integral characteristics 
of the homogeneous condensation process (the mass fraction of the condensed phase and the amount of condensation heat released into the flow), which leads to good agreement between the results of calculations and experiments.

\subsection{Heterogeneous Condensation of Water Vapor in a Shock Tube}

The proposed mathematical model and the developed computational algorithm were verified by comparing the computational results with the experimental data acquired by Smolders [28]. In this experiment, carried out in a double-diaphragm shock tube, both heterogeneous condensation and the evaporation of water vapor on nanometer particles were considered. In the experiment, the measurements of pressure, temperature, level of supersaturation, and average radius of droplets at the control point were carried out at different times.

The experimental setup (Figure 6) consisted of a high-pressure chamber (HPC), a lowpressure pipe (LPP) that was $12.8 \mathrm{~m}$ long, a vacuum chamber (VC), and an experimental point (EP). The behavior of a gas-droplet mixture with solid impurities in the LPP was considered. The HPC was filled with pure nitrogen at a pressure of 1.9 bar. The PLL was filled with a mixture of nitrogen, water vapor, and an admixture of solid particles. The nitrogen pressure was $p=0.997$ bar, the water vapor pressure was $p_{v}=2090 \mathrm{~Pa}$, the particle radius was $r_{n}=15 \mathrm{~nm}$, and their concentration was $n_{n}=2 \times 10^{12} \mathrm{~m}^{-3}$. The temperature in all parts of the experimental setup was $294.3^{\circ} \mathrm{K}$.

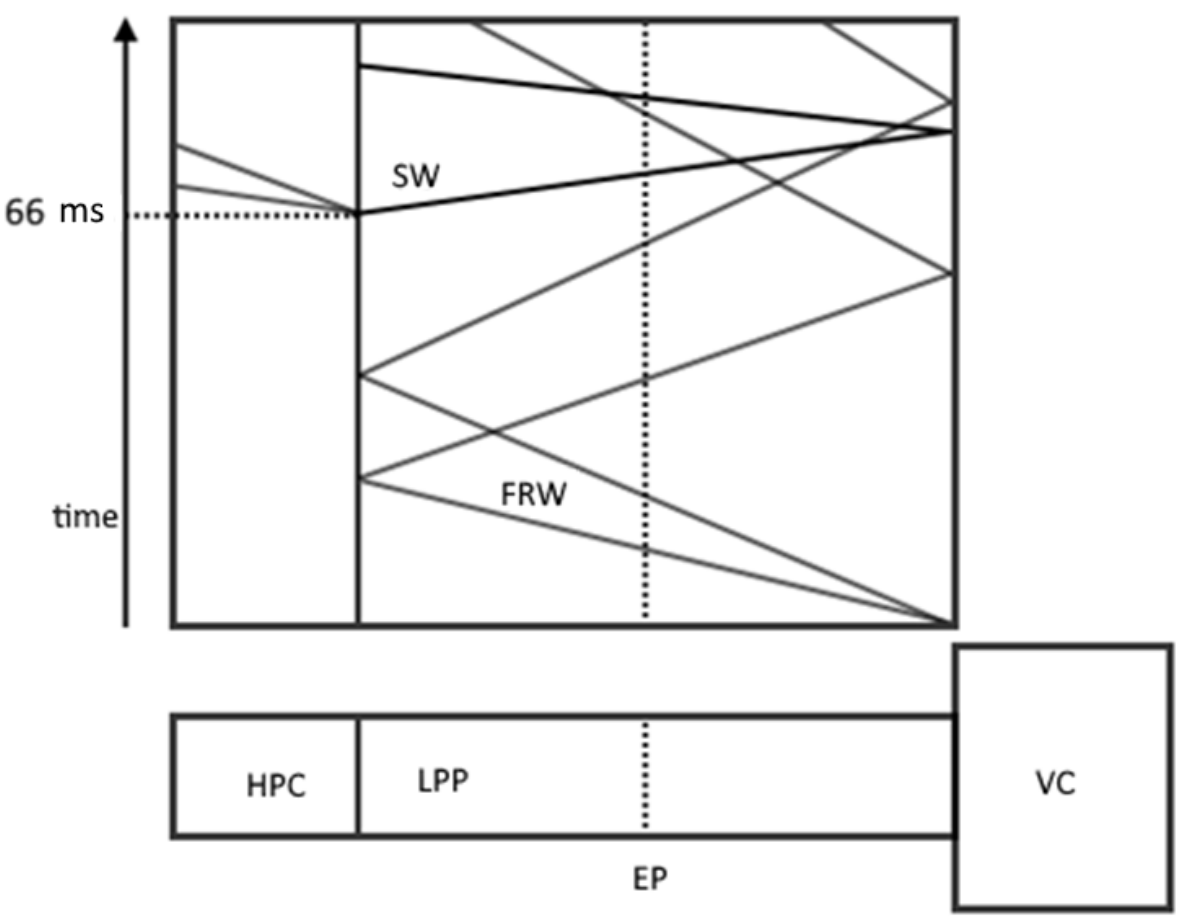

Figure 6. Schematic representation of the experimental setup and $x$ - $t$ diagram of the process. FRW is a fan of rarefaction waves; SW is a shock wave.

At the beginning of the experiment, the VC was opened and the necessary pressure was maintained by special pumps so that the gas flow had a Mach number of 0.212 . After that, the HPC opened at $66 \mu \mathrm{s}$, which generated the propagation of a shock wave along the LPP, forcing the complete evaporation of water droplets.

As a computational domain, a long pipe was considered, which had three regions of $12.8 \mathrm{~m}$ each, which played the role of HPC, LPP, and VC (Figure 7). EP was located at a distance of $6.17 \mathrm{~m}$ from the line of separation of the HPC and LPP. 


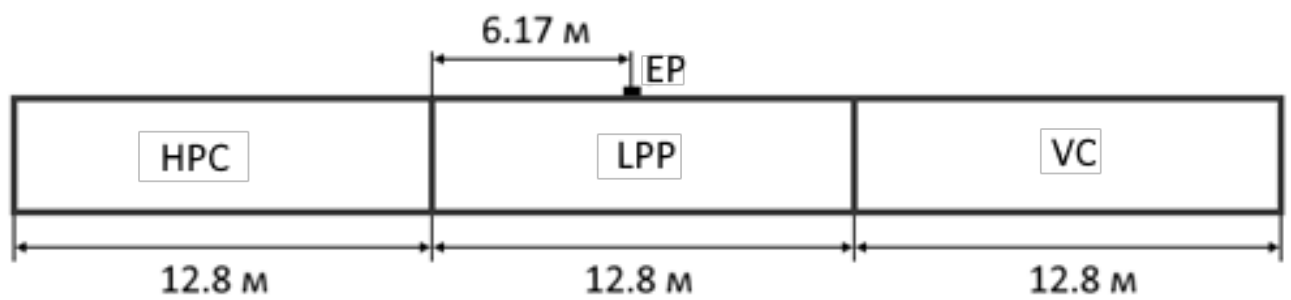

Figure 7. Geometrical parameters of the computational region.

At $66 \mathrm{~ms}$ of the experiment, a rupture of the diaphragm separating the HPC and LPT occurred. The left boundary was a solid wall. Free boundary conditions were used on the right boundary of the VC. The initial parameters in each region, according to [28], are given in Table 1.

Table 1. The initial parameters in each region.

\begin{tabular}{cccc}
\hline Parameter & HPC & LPP & VC \\
\hline pressure (bar) & 1.9 & 0.99909 & 0.565 \\
temperature (K) & 293.4 & 293.4 & 293.4 \\
$\alpha_{\max }$ & $1 \times 10^{-7}$ & 0.013297 & 0.013297 \\
\hline
\end{tabular}

To obtain a Mach number of 0.212 at the interface between the LPP and VC, a pressure of 0.565 bar was set in the VC [26]. To ensure the stability of the calculation, it was assumed that the HPC contained water vapor in a very low concentration. The size of the radii of solid particles varied from $r_{\min }^{s}=10^{-8} \mathrm{~m}$ to $r_{\max }^{s}=2 \times 10^{-8} \mathrm{~m}$.

The numerical calculation was carried out on a one-dimensional grid consisting of 2000 cells. Figure 8 shows the calculation results in the form of the time dependence of the flow parameters at the experimental point from the beginning of the process up to the time instant of $90 \mathrm{~ms}$. For pressure (Figure 8a), good agreement of the numerical results (solid blue line) with the experimental results (red circles) is seen. Figure 8d shows that the calculated values of the saturation ratio are somewhat larger than the experimental ones in the interval from $20 \mathrm{~ms}$ to $40 \mathrm{~ms}$. The average size of liquid droplets also differs from the experimental values, starting from $40 \mathrm{~ms}$ (Figure $8 \mathrm{c}$ ), by $5 \%$. Analysis of the calculation results shown in Figures $8 \mathrm{a}-\mathrm{d}$ and 9 shows that the rarefaction wave from the $\mathrm{VK}$, propagating to the left, reaches the EP at the time $t=19 \mathrm{~ms}$. At $t=20 \mathrm{~ms}$, condensation begins at this point, and at $t=25 \mathrm{~ms}$, a condensation shock appears as a result of the thermal effect of condensation (Figure 8a). At time $t=52.5 \mathrm{~ms}$, the rarefaction wave reflected from the left boundary of the LPP arrives at EP, and then at $t=64 \mathrm{~ms}$ the secondary condensation shock arrives at EP as well (Figures $8 \mathrm{a}-\mathrm{c}$ and 9). At $66 \mathrm{~ms}$, as a result of the rupture of the diaphragm between HPC and LPP, a shock wave is formed, which propagates to the right and reaches the EP at time $t=80 \mathrm{~ms}$. A sharp increase in temperature behind the shock wave front (Figure 8 b) leads to a decrease in saturation below unity (Figure $8 \mathrm{~d}$ ), the evaporation of heterogeneous droplets and, consequently, a decrease in the average droplet radius (Figure $8 \mathrm{c}$ ) to the radius of solid particles. Figure $8 \mathrm{~b}$ shows that the temperature jump behind the shock wave is $2 \%$ less than the experimental one.

Figure 9 shows that condensation creates an additional density jump in the LPP region.

The use of the assumption of instantaneous wetting in the model of heterogeneous condensation allows one to obtain adequate results when simulating the experiment [28]. The above comparison with the experiment in a shock tube confirms the possibility of using the developed mathematical model and numerical algorithm for modeling heterogeneous condensation. 


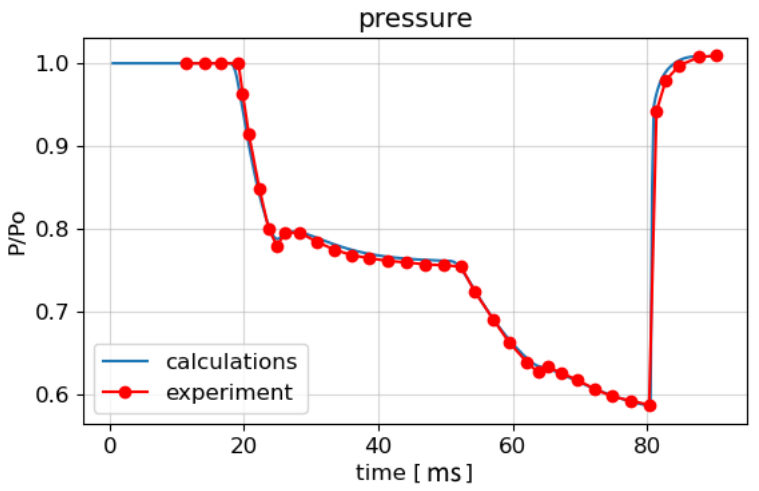

(a)

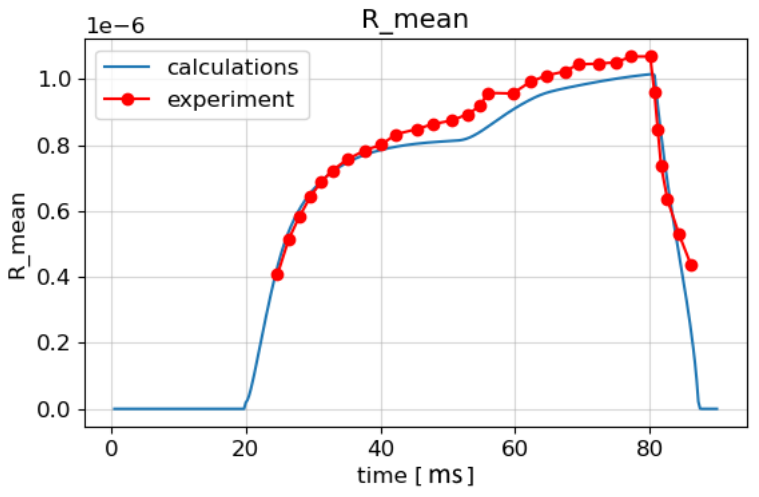

(c)

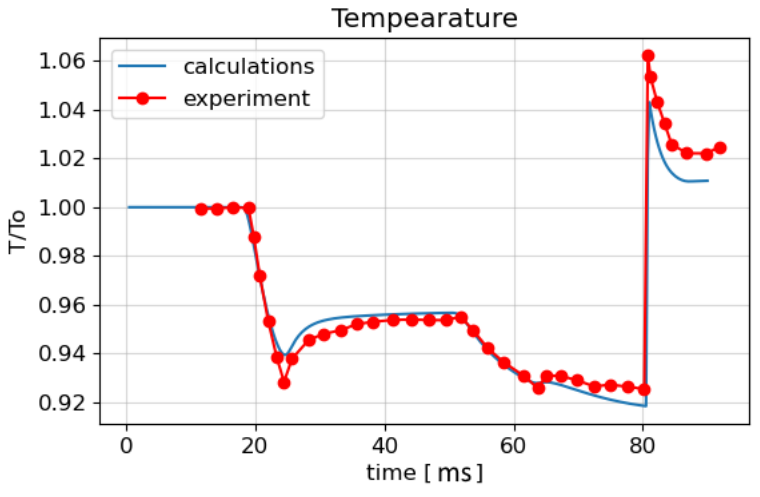

(b)

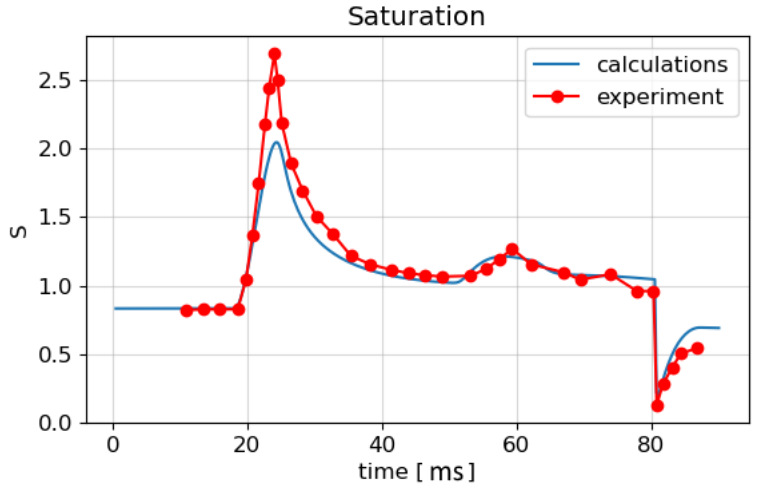

(d)

Figure 8. Comparison of experimental results [28] and calculations: (a) the pressure; (b) the temperature; (c) the average particle radius; (d) the saturation ratio.

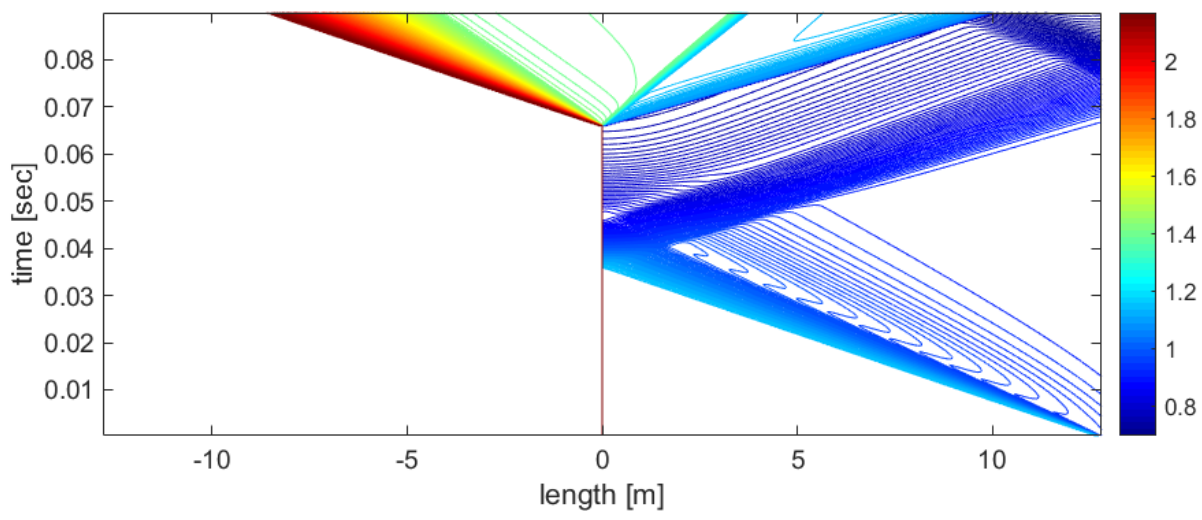

Figure 9. $x-t$ diagram of the process, visualized using the density contour lines, for the HPC and LPP regions.

\section{Numerical Results}

Using the developed algorithm, the numerical simulation of gas-dynamic flows with phase transitions in an ejector device for cleaning smoky gases is carried out. An overview of various ways of using ejectors is given in [45]. The geometry of the calculated device consists of two coaxially located conical nozzles, as shown in Figure 10. The corresponding dimensions are given in Table 2. Water vapor comes from the area designated in_1 in the first nozzle and ejects air from the in_2 area of the second nozzle (Figure 10). 


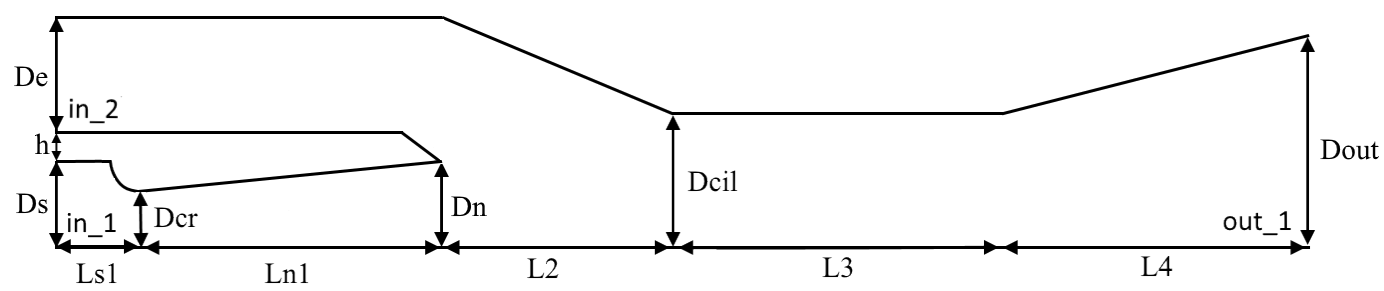

Figure 10. The geometry of the ejection filtration device.

Table 2. Ejector device geometry.

\begin{tabular}{cc}
\hline Parameter & Value, $\mathbf{m m}$ \\
\hline$D_{e}$ & 4.0 \\
$D_{s}$ & 4.3 \\
$D_{c r}$ & 3.0 \\
$D_{n}$ & 3.5 \\
$D_{\text {cil }}$ & 5.5 \\
$D_{\text {out }}$ & 7.0 \\
$h$ & 0.5 \\
$L_{s 1}$ & 20 \\
$L_{n 1}$ & 60 \\
$L_{2}$ & 50 \\
$L_{3}$ & 250 \\
$L_{4}$ & 80 \\
\hline
\end{tabular}

In the numerical simulation, a block-structured computational grid is used. The grid has been adapted to the diffuser wall and the upper nozzle wall. The grid consists of about seventy thousand quadrangular cells.

We assume that the flow is turbulent. The algebraic turbulence model [41] is used to calculate the turbulent viscosity and thermal conductivity.

The pressure and temperature values are set as input conditions at the boundaries in_1 and in_2. Constant pressure is set in the outer area behind the nozzle out_1. Values of temperatures and pressures are given in Table 3 . The maximum concentration of solid particles of $50 \mathrm{~nm}$ in size was 0.005 .

Table 3. The boundary conditions.

\begin{tabular}{ccc}
\hline Boundary & Pressure, Pa & Temperature, K \\
\hline in_1 & $8.0 \times 10^{5}$ & 437 \\
in_2 & $1.75 \times 10^{5}$ & 350 \\
out_1 & $1.0 \times 10^{5}$ & - \\
\hline
\end{tabular}

For efficient operation of the device, it is necessary that the flows of wet steam from the first nozzle and the ejected gas at the inlet to the second nozzle mix well in the mixing zone of the second nozzle, and as much condensate as possible should form in this zone. Therefore, to improve mixing in this system, a very long cylindrical part of the second nozzle is chosen. The first nozzle has a small degree of geometric expansion to prevent the formation of a large amount of condensate inside the nozzle.

To estimate the possible amount of condensate in the system, numerical simulation of the ejector operation was carried out for the case in which there are no foreign particles in the system and only homogeneous condensation occurs. The numerical calculation established the existence of three regions inside the ejector, where water condensate is actively formed. The first region is located inside the first nozzle, the second region is located directly behind the first nozzle, and the third region is in the diffuser of the second nozzle. In this case, the mass fraction of water condensate in the diffuser of the second 
nozzle reaches a value of 0.11 . In numerical experiments, the ratio of injected gas to water vapor was about $25 \%$ at a water flow rate of about $120 \mathrm{~L}$ per hour. With the considered parameters of the problem, there were no zones of active evaporation of water droplets in the ejector.

The results of numerical calculations show that there is no heterogeneous condensation up to the critical section of the second nozzle. However, as a result of mixing in the diffuser, it causes the appearance of heterogeneous condensation in the second nozzle. However, due to an insufficient degree of mixing (Figures 11 and 12) and a low level of supersaturation in the second nozzle, heterogeneous condensation occurs much less intensively than homogeneous condensation.

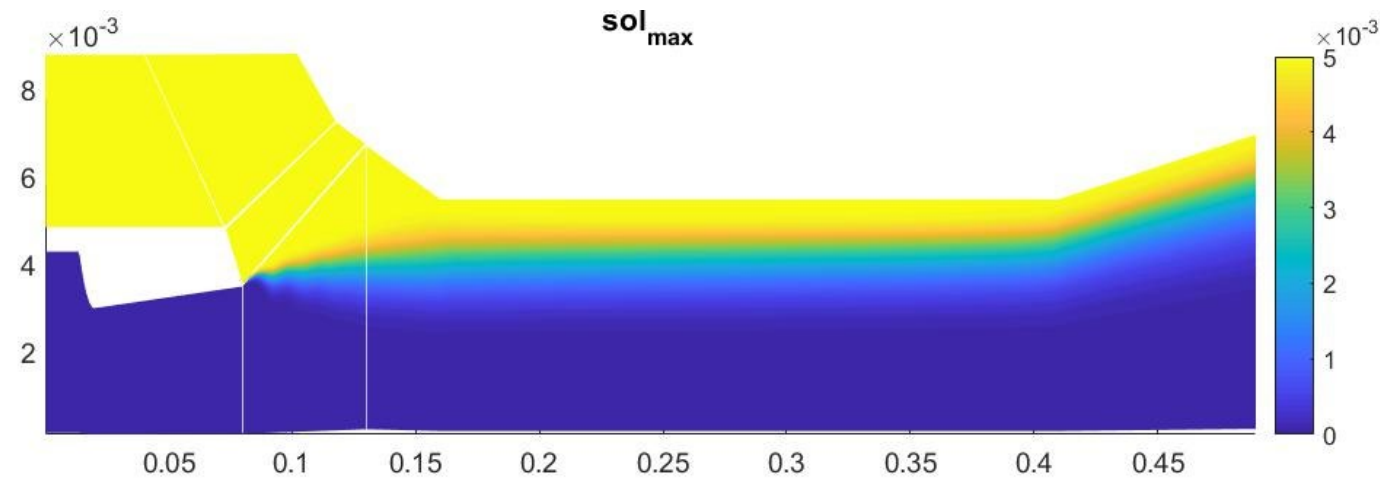

Figure 11. Solid particle concentration distribution.

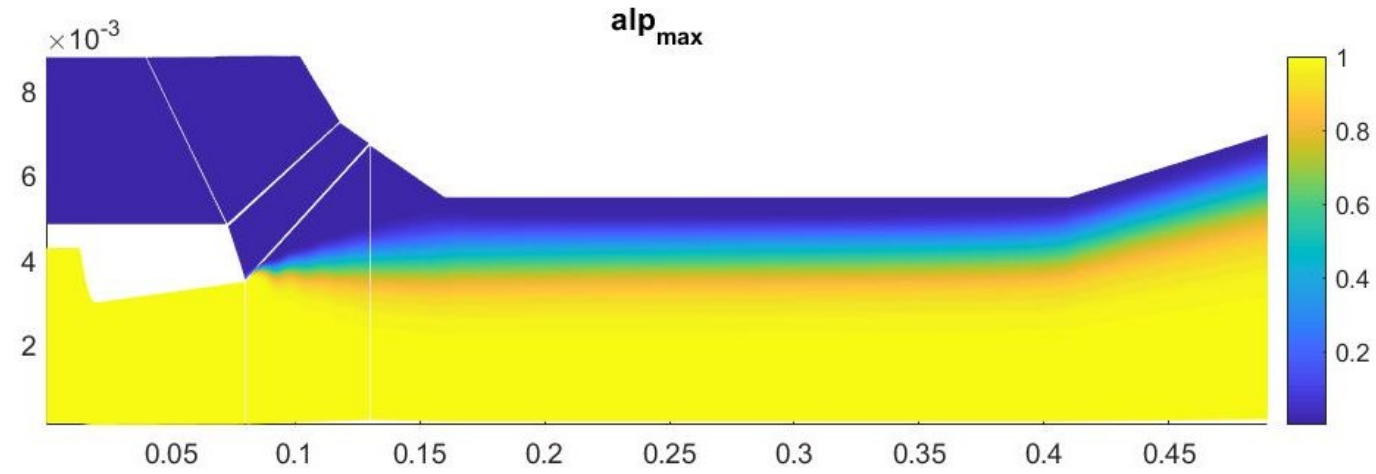

Figure 12. Distribution of the mass fraction of the condensable substance.

The amount of condensate on the axis of symmetry in this case is about $13 \%$ (Figure 13). Most of the heterogeneous condensation occurs in the mixing region near the wall of the second nozzle (Figure 14). In the near-wall region, condensation occurs weakly due to an increase in temperature in the boundary layer.

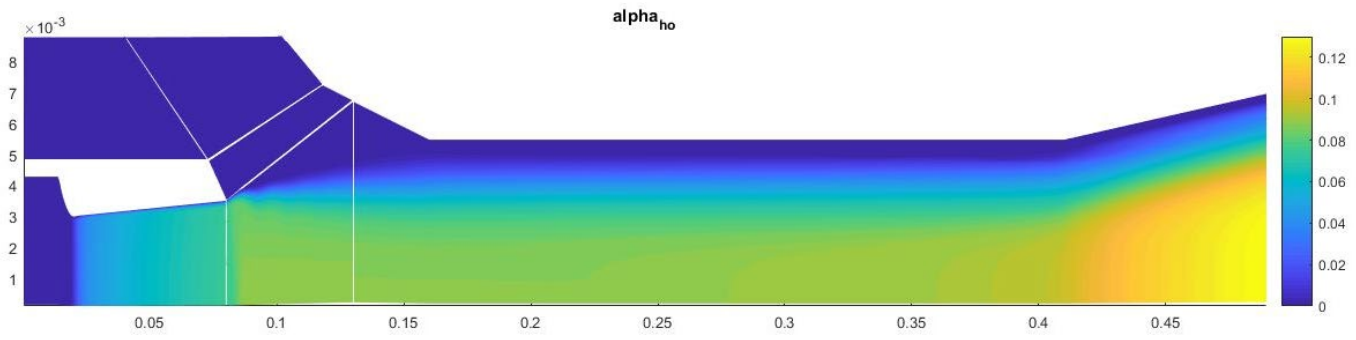

Figure 13. Contours of the field of the mass fraction of water formed in homogeneous condensation. 


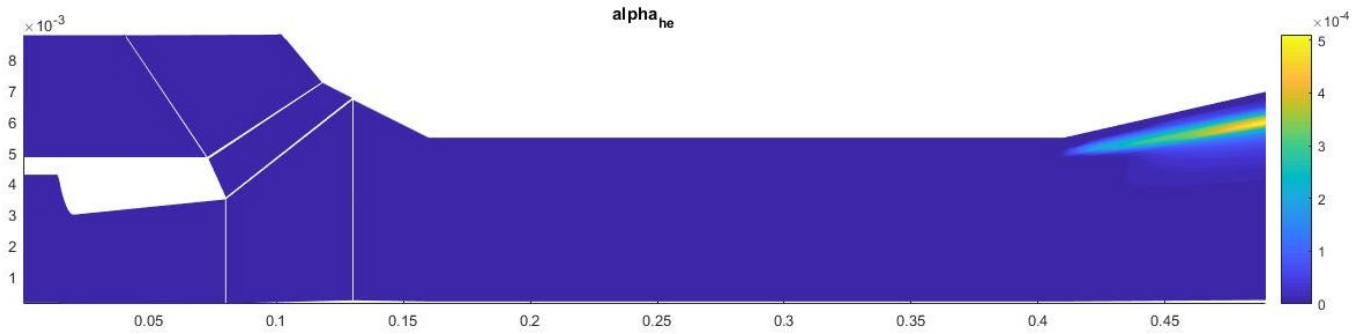

Figure 14. Contours of the field of the mass fraction of water formed in heterogeneous condensation.

A study of the parameters at the exit from the second nozzle shows that in this device the number of homogeneous clusters is much higher than that of heterogeneous ones, which prevents the greater growth of heterogeneous droplets. This leads to the fact that the increase in the total volume of homogeneous droplets is higher than that of heterogeneous ones, at a lower growth rate of the droplet. Under such conditions, heterogeneous condensation is weakly manifested, which does not allow droplets on solid particles to grow significantly in size (Figures 15-17).

The contribution of heterogeneous condensation to the flow turns out to be insignificant. This is due to the small number of heterogeneous clusters. There are several orders of magnitude more homogeneous clusters. This leads to the fact that a small increase in homogeneous droplets causes a significantly greater increase in the amount of $\alpha^{\text {ho }}$ than for $\alpha^{\text {he }}$ of heterogeneous wetted particles. The growth rate of heterogeneous clusters is insufficient to significantly increase the volume of water on the particles, and therefore the heterogeneous particles are too small for their further separation using centrifugal separators. However, coating the particles with water can increase the efficiency of electrostatic filters. An electric charge can cause even the smallest droplets to settle when using electric fields.

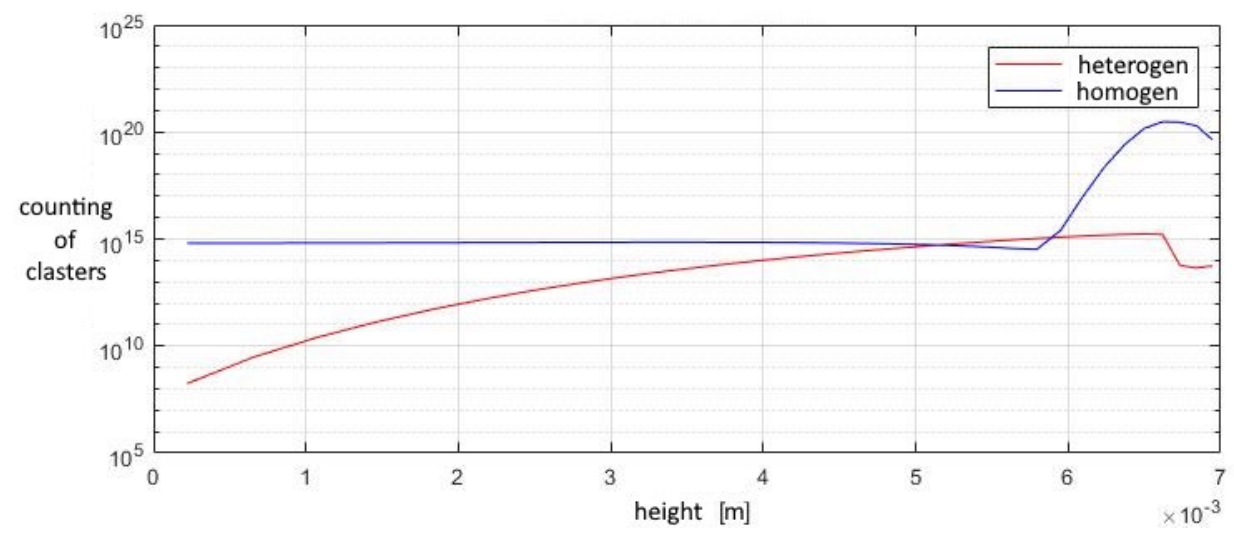

Figure 15. The number of clusters formed in the outlet section of the diffuser.

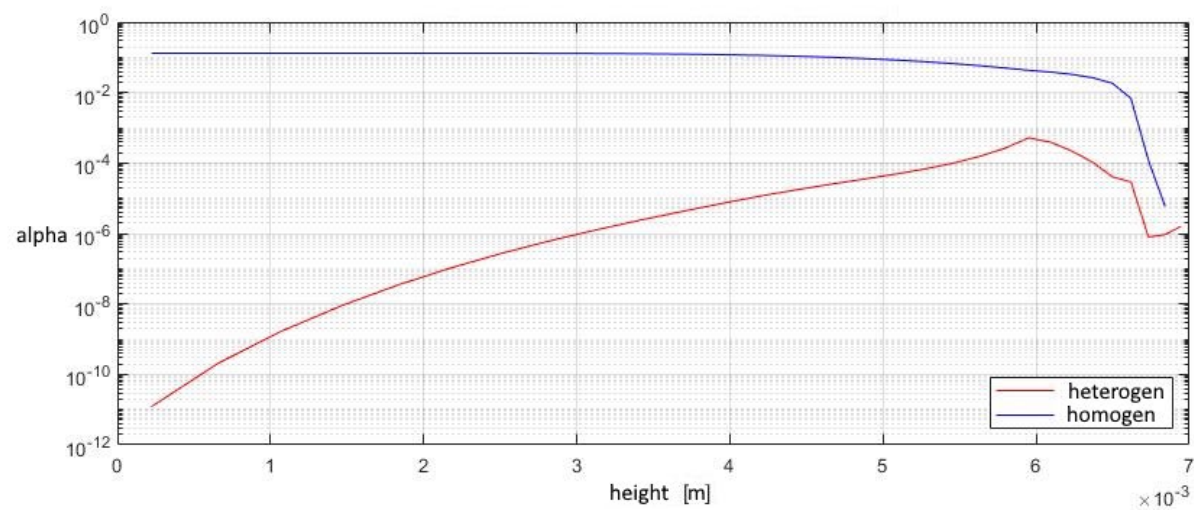

Figure 16. The mass fraction of condensed substance in the outlet section of the diffuser. 


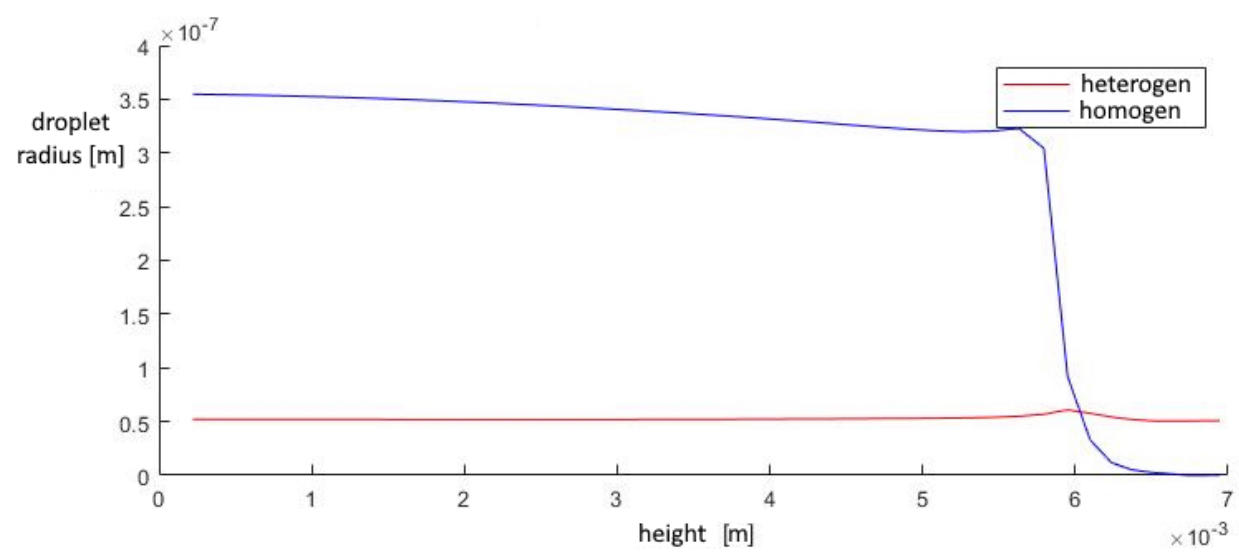

Figure 17. The average droplet size in the outlet section of the diffuser.

\section{Conclusions}

In this paper, we have proposed a mathematical model for the study of gas-dynamic multiphase flows with the simultaneous occurrence of phase transition processes.

An approach based on the use of the moment method for modeling homogeneous and heterogeneous condensation and evaporation is considered.

The system of equations for describing unsteady gas-dynamic processes based on the Navier-Stokes equations and equations of the method of moments for the phase transition processes in two-dimensional plane and axisymmetric cases has been expanded by adding two equations for the mass fractions of the condensing substance and the solid particles. This allows us to significantly extend the class of problems to be solved.

Using the developed algorithm, a numerical simulation of gas-dynamic flow with phase transitions in an ejector device for cleaning smoky gases has been carried out. Superheated steam is supplied to the inlet of the first nozzle and condenses in the supersonic part of the nozzle. A two-phase vapor-droplet mixture ejects dusty gas from the second nozzle and mixes with it. In the expanding part of the second nozzle of the ejector, heterogeneous condensation occurs on dust particles, which increases their mass. In the considered case, the size of the particles increases insignificantly, which prevents the separation of these particles due to centrifugal force. However, wetted particles can increase the efficiency of other cleaning methods, one example of which is an electrostatic filter.

Author Contributions: Conceptualization, I.E.I. and V.S.N.; methodology, I.A.K., I.E.I. and V.S.N.; software, V.S.N.; validation, I.A.K., I.E.I. and V.S.N.; formal analysis, I.A.K.; investigation, I.E.I. and V.S.N.; resources, I.E.I. and V.S.N.; data curation, I.A.K.; writing-original draft preparation, I.A.K. and I.E.I.; writing-review and editing, I.E.I.; visualization, V.S.N.; supervision, I.A.K.; project administration, I.E.I. All authors have read and agreed to the published version of the manuscript.

Funding: This research received no external funding.

Acknowledgments: Special thanks to Nikolai Yuryevich Bykov, leading researcher of Peter the Great St. Petersburg Polytechnic University and Arseny Konstantinovich Yastrebov, senior researcher of the National Research University "Moscow Power Engineering Institute" for valuable comments in the preparation of the work.

Conflicts of Interest: The authors declare no conflict of interest.

\section{References}

1. Wyslouzil, B.E.; Wölk, J. Overview: Homogeneous nucleation from the vapor phase-The experimental science. J. Chem. Phys. 2016, 145, 211702. [CrossRef] [PubMed]

2. Anisimova, M.P.; Fominykh, E.G.; Akimov, S.V.; Hopke, P.K. Vapor-gas/liquid nucleation experiments: A review of the challenges. Aerosol Sci. 2009, 40, 733-746. [CrossRef]

3. Muitjens, M.J.E.H. Homogeneous Condensation in a Vapour/Gas Mixture at High Pressures in an Expansion Cloud Chamber. Ph.D. Thesis, Technische Universiteit Eindhoven, Eindhoven, The Netherlands, 1996. [CrossRef] 
4. Kalikmanov, V.I. Nucleation Theory; Springer: Dordercht, The Netherlands, 2013. [CrossRef]

5. Manka, A.A.; Brus, D.; Hyvärinen, A.-P.; Lihavainen, H.; Wölk, J.; Strey, R. Homogeneous water nucleation in a laminar flow diffusion chamber. J. Chem. Phys. 2010, 132, 244505. [CrossRef] [PubMed]

6. Brus, D.; Ždímal, V.; Smolík, J. Homogeneous nucleation rate measurements in supersaturated water vapor I. J. Chem. Phys. 2008, 129, 174501. [CrossRef]

7. Brus, D.; Ždímal, V.; Smolík, J. Homogeneous nucleation rate measurements in supersaturated water vapor II. J. Chem. Phys. 2009, 131, 074507. [CrossRef] [PubMed]

8. Mayorov, V.O.; Yastrebov, A.K.; Levashov, V.Y. Application of the homogenous nucleation theory to the study of explosive boiling of superheated liquid drops. J. Phys. Conf. Ser. 2020, 1683, 022019. [CrossRef]

9. Bykov, N.Y.; Gorbachev, Y.E. Cluster formation in copper vapor jet expanding into vacuum: The direct simulation Monte Carlo. Vacuum 2019, 163, 119-127. [CrossRef]

10. Kuni, F.M.; Shchekin, A.K.; Grinin, A.P. Theory of heterogeneous nucleation for vapour undergoing a gradual metastable state formation process. Adv. Phys. Sci. 2001, 44, 331-370. [CrossRef]

11. Jurski, K.; Gehin, E. Heterogeneous condensation process in an air water vapour expansion through a nozzle-experimental aspect. Int. J. Multiph. Flow 2003, 29, 1137-1152. [CrossRef]

12. Fan, Y.; Qin, F.; Luo, X.; Lin, L.; Gui, H.; Liu, J. Heterogeneous condensation on insoluble spherical particles: Modeling and parametric study. Chem. Eng. Sci. 2013, 102, 387-396. [CrossRef]

13. Xu, J.; Yu, Y.; Zhang, J.; Meng, Q.; Zhong, H. Heterogeneous condensation of water vapor on particles at high concentration. Powder Technol. 2017, 305, 71-77. [CrossRef]

14. Xu, J.; Yu, Y.; Yin, Y.; Zhang, J.; Zhong, H. Heterogeneous condensation coupled with partial gas circulation for fine particles abatement. Chem. Eng. J. 2017, 330, 979-986. [CrossRef]

15. Lee, D.-W.; Hopke, P.K.; Rasmussen, D.H.; Wang, H.-C.; Mavliev, R. Comparison of Experimental and Theoretical Heterogeneous Nucleation on Ultrafine Carbon Particles. J. Phys. Chem. B 2003, 107, 13813-13822. [CrossRef] [PubMed]

16. Lv, L.; Zhang, J.; Xu, J.; Yi, J. Heterogeneous condensation process observed by environmental scanning electron microscopy (ESEM): On smooth single aerosol particle. Aerosol Sci. Technol. 2020, 54, 1515-1526. [CrossRef]

17. Van Putten, D.S.; Sidin, R.S.R.; Hagmeijer, R. Efficient approximation of the cluster size distribution in binary condensation. J. Chem. Phys. 2010, 132, 184511. [CrossRef]

18. Hill, P.G. Condensation of water vapour during supersonic expansion in nozzles. J. Fluid Mech. 1966, 25, 593-620. [CrossRef]

19. Luo, X.; Lamanna, G.; Holten., A.P.C.; van Dongen, M.E.H. Effects of homogeneous condensation in compressible flows: Ludwieg-tube experiments and simulations. J. Fluid Mech. 2007, 572, 339-366. [CrossRef]

20. Hagmeijer, R.; IJzermans, R.H.A.; Put, F. Solution of the general dynamic equation along approximate fluid trajectories generated by the method of moments. Phys. Fluids 2005, 17, 056101. [CrossRef]

21. McGraw, R. Description of aerosol dynamics by the quadrature method of moments. Aerosol Sci. Technol. 1997, 27, 255-265. [CrossRef]

22. Alam, M.M.D.A.; Takao, M.; Setoguchi, T. Numerical Simulation of Non-Equilibrium Two-Phase Wet Steam Flow through an Asymmetric Nozzle. Fluids 2017, 2, 63. [CrossRef]

23. Gidaspov, V.; Ivanov, I.; Nazarov, V.; Malashin, F.; Kryukov, I. Study of the condensation process in nozzles with a large degree of expansion. Phys. Chem. Kinet. Gas Dyn. 2018, 19, 1-17. [CrossRef]

24. Nazarov, V.S.; Ivanov, I.E.; Kryukov, I.A. Numerical study of gas-dynamic and condensation processes in an ejector device which used for cleaning gas-air emissions. IOP Conf. Ser. Mater. Sci. Eng. 2020, 927, 012063. [CrossRef]

25. Nazarov, V.S.; Ivanov, I.E.; Kryukov, I.A. The Investigation of the Evolution of Cluster Beam Development in the Nozzle-Skimmer System. Smart Innov. Syst. Technol. 2020, 217, 69-85.

26. Luo, X.; Cao, Y.; Xie, H.; Qin, F. Moment method for unsteady flows with heterogeneous condensation. Comput. Fluids. 2017, 146, 51-58. [CrossRef]

27. Matsuo, S.; Tanaka, M.; Alam, M.M.D.A.; Alam, M.D.M.; Setoguchi, T. Numerical study of heterogeneous condensation in the transonic flow fields. In Proceedings of the International Conference on Mechanical Engineering 2005 (ICME2005), Dhaka, Bangladesh, 28-30 December 2005; ICME05-FL-19.

28. Smolders, H.J.; Nissen, E.M.J.; van Dongen, M.E.H. The random choice method applied to non-linear wave propagation in gas-vapour-droplets mixtures. Comput. Fluids 1992, 21, 63-75. [CrossRef]

29. Liu, X.Y. Heterogeneous nucleation or homogeneous nucleation. J. Chem. Phys. 2000, 112, 9949-9955. [CrossRef]

30. Maattanen, A.; Vehkamaki, H.; Lauri, A.; Merikallio, S.; Kauhanen, J.; Savijarvi, H.; Kulmala, M. Nucleation studies in the Martian atmosphere. J. Geophys. Res. 2005, 110, E02002. [CrossRef]

31. Frenkel, J. Kinetic Theory of Liquids; Oxford University Press: New York, NY, USA, 1946.

32. Becker, R.; Döring, W. Kinetische behandlung der Keimbildung in ubersattingten damfen. Annln Physik 1935, $24,719-752$. [CrossRef]

33. Zeldovich, J. Theory of the formation of a new phase. J. Expl. Theoret. Phys. 1942, 12, 525.

34. Fletcher, N.H. Size Effect in Heterogeneous Nucleation. J. Chem. Phys. 1958, 29, 572-576. [CrossRef]

35. Yuan, C.; Fox, R.O. Conditional quadrature method of moments for kinetic equations. J. Comput. Phys. 2011, $230,8216-8246$. [CrossRef] 
36. Gerber, A.G.; Mousavi, A. Application of quadrature method of moments to the polydispersed droplet spectrum in transonic steam flows with primary and secondary nucleation. Appl. Math. Model. 2007, 31, 1518-1533. [CrossRef]

37. Gerber, A.G.; Mousavi, A. Representing polydispersed droplet behavior in Nucleating Steam Flow. J. Fluids Eng. 2007, 129, 1404-1414. [CrossRef]

38. Luo, X. Unsteady Flows with Phase Transition. Ph.D. Thesis, Technische Universiteit Eindhoven, Eindhoven, The Netherlands, 2004. [CrossRef]

39. Luo, X.; Bart, P.; van Dongen, M.E.H.; Hoeijmakers, H.W.M.; Yang, J. On phase transition in compressible flows: Modelling and validation. J. Fluid Mech. 2006, 548, 403-430. [CrossRef]

40. Kortsenshteyn, N.M.; Yastrebov, A.K. Effect of interphase heat transfer on bulk condensation in dust-laden vapor-gas flow. J. Phys. Conf. Ser. 2020, 1683, 022062. [CrossRef]

41. Camelli, F.; Loehner, R. Combining the Baldwin Lomax and Smagorinsky turbulence models to calculate flows with separation regions. In Proceedings of the 40th AIAA Aerospace Sciences Meeting \& Exhibit, Reno, NV, USA, 14-17 January 2002. [CrossRef]

42. Gyarmathy, G. The spherical droplet in gaseous carrier streams: Review and synthesis. In Multiphase Science and Technology; Springer: Berlin/Heidelberg, Germany, 1982; Volume 1.

43. Wyslouzil, B.E.; Heath, C.H.; Cheung, J.L.; Wilemski, G. Binary condensation in a supersonic nozzle. J. Chem. Phys. 2000, 113, 7317-7329. [CrossRef]

44. Gyarmathy, G. Zur Wachstumsgeschwindigkeit kleiner Flussigkeitstropfen in einer ubersattigten Atmosphare. Z. Angew. Math Phys. 1963, 14, 280-293. [CrossRef]

45. Koirala, R.; Ve, Q.L.; Zhu, B.; Inthavong, K.; Date, A. A Review on Process and Practices in Operation and Design Modification of Ejectors. Fluids 2021, 6, 409. [CrossRef] 